que reafirma la capacidad de naturalización del táxon en el territorio valenciano.

\section{BIBLIOGRAFÍA}

BADRÉ, F. y R. DESCHÀTRES -1979- LeS ptéridophytes de la France. Liste commentée des espèces (taxonomie, cytologie, écologie et répartition générale). Candollea 34: 379-457.

CARRETERO, J.L. y A. AGUILELLA - 1995- Flora y Vegetación nitrófilas del término municipal de la ciudad de Valencia. Ajuntament de València.

CASSASAYAS, T. y A. FARRÀS -1986- Polystichum falcatum (L. fil.) Diels, adventícia a Catalunya. Collectanea Botanica 16(2): 425-426.

HERRERO-BORGOÑÓN, J.J., I. MARTÍNESSOLÍS, E. ESTRELLES y A.M. IBARS - 1997 Avance al atlas pteridológico de la Comunidad Valenciana. Flora montiberica 7: 72-86.
IBARS, A.M, J.J. HERRERO-BORGOÑÓN, E. ESTRELLES y I. MARTÍNEZ-SOLÍS -1999Helechos de la Comunidad Valenciana. Colección Biodiversidad 6: 1-163.

SAMO, A.J. -1995- Catálogo florístico de la provincia de Castellón. Diputació de Castelló. SIMÕES DA SILVA, A. y J. DOMINGUES DE ALMEIDA -2000- Nephrolepis exaltata (Nephrolepidaceae), naturalized in Europe. Anales Jard. Bot. Madrid 58(1): 182.

Aceptado para su publicación en abril de 2001

Dirección del autor. Departamento de Agricultura y Economía Agraria, Facultad de Veterinaria, Universidad de Zaragoza. C/ Miguel Servet, 177. E50013 Zaragoza. E-mail: jogasemo@posta.unizar.es

\title{
50. NUEVAS LOCALIDADES DE OPHIOGLOSSUM VULGATUM L. EN LA COMUNIDAD VALENCIANA
}

\author{
José Vicente ANDRÉS ROS, Antonio José CASTELLÓ MONSORIU y \\ Nieves SARASA ALCUBIERRE
}

New localities of Ophioglossum vulgatum L. in the Community of Valencia.

Palabras clave. Corología, Ophioglossaceae, Ophioglossum vulgatum, Castellón, Comunidad Valenciana.

Key words. Chorology, Ophioglossaceae, Ophioglossum vulgatum, Castellón, Community of Valencia.

CASTELLÓN: Alto Palancia, Bejís, Sierra de El Toro, entre Bco. de la Marina y Bco. del Rasinero, 30SXK9019, $1200 \mathrm{~m}, 1$-VII-2000, J. Andrés, N. Sarasa \& T. Castelló, VAL 117811;
Alto Palancia, El Toro, Sierra de El Toro, Bco. de la Musa, 30SXK8823, $1180 \mathrm{~m}, 2$-VII-2000, J. Andrés, N. Sarasa \& T. Castelló, VAL 117812. 


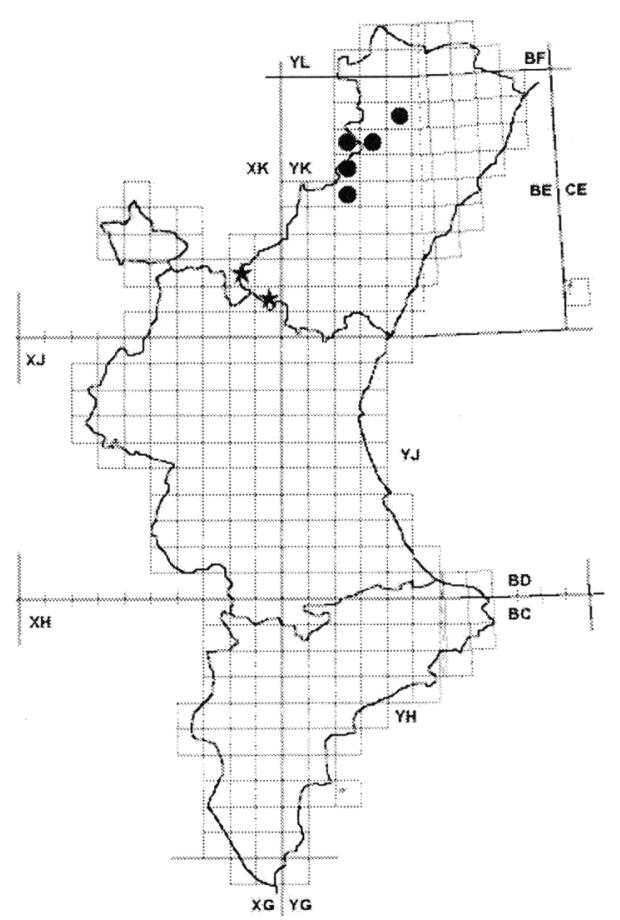

Figura 1: Distribución de Ophioglossum vulgatum L. en la Comunidad Valenciana, en cuadrículas UTM de $10 \times 10 \mathrm{~km}$ : (๑) localidades conocidas; $(\star)$ localidades nuevas.

Ophioglossum vulgatum L. es un pteridófito ampliamente distribuido por el hemisferio norte. En la Península Ibérica está presente, fundamentalmente, en la mitad norte y oeste, aunque también se localiza en los Sistemas Bético y Penibético, (Salvo et al., 1984; Castroviejo et al., 1986; Carrión et al., 2000).

En la Comunidad Valenciana, únicamente se conocía en cinco localidades de la provincia de Castellón (cf. Ibars et al., 1999), y se considera una especie muy rara, catalogada "en peligro" (EN), de acuerdo con las categorías propuestas por la UICN.

En 1867, Salvador (1867) la encontró en dos localidades: una dentro del término municipal de Ares del Maestre (30TYK48) y otra en el término de Villafranca (30TYK37). Posteriormente, en 1968, Vigo (1968) la localizó en cuatro puntos del macizo de Peñagolosa: sobre la Font de la Pegunta, Benages (ambas en la cuadrícula UTM 30TYK25), El Rebollar y Maset del Zurdo (estas últimas en la cuadrícula UTM 30TYK26).

Desde 1867, Ophiglossum vulgatum no ha vuelto a ser observado en las localidades señaladas por J. Salvador, por lo que Ibars et al. (l.c.) consideran dudosa su actual presencia en ellas, presumiendo su desaparición. No obstante, en 1993, C. Fabregat y S. López Udias la encontraron en los alrededores de Villafranca, en la cuadrícula UTM 30TYK27 (VAB 945042), contigua a la de J. Salvador (Fabregat, 1995).

Las nuevas localidades amplían su distribución hacia el sur de la provincia de Castellón y refuerzan su presencia en el este peninsular (fig. 1).

Las poblaciones encontradas se sitúan, mayoritariamente, en pastizales y juncales muy húmedos o encharcados, pertenecientes a la clase Molinio-Arrhenatheretea R. Tuxen 1937. En ambas localidades, aparece junto a especies tales como Scirpus holoschoenus L., Dactylorhiza elata (Poir.) Soó, Cirsium monspesulanum subsp. ferox (Cos.) Talavera y Trifolum pratense L., entre otras. Sin embargo, en la localidad de El Toro, algunos individuos fueron hallados en una chopera de Populus nigra L., temporalmente encharcada, así como en un herbazal algo más seco, junto a Genista scorpius (L.) DC., Trifolium pratense, Genista hispanica L., Hypericum perforatum L. y Scirpus holoschoenus, entre otras.

Hay que destacar la magnitud de las dos poblaciones, las cuales contaban con unos 350 pies en la localidad Bejís y, en torno a los 1.000 pies, en la localidad de El Toro. 
AGRADECIMIENTOS. Queremos expresar nuestro sincero agradecimiento a J. Güemes por su ofrecimiento a la revisión de la presente nota. No queremos dejar de nombrar a S. López Udias, C. Fabregat, J. Riera, A. Ibars, E. Estrelles, y J. J. Herrero-Borgoñón, por el apoyo y/o la ayuda desinteresada que nos han of recido en todo momento. Estas observaciones resultan del estudio Aportaciones al conocimiento de las orquídeas del Alto Palancia, subvencionado, en 1999, por la Consellería de Medio Ambiente de la Generalitat Valenciana.

\section{BIBLIOGRAFÍA}

CARRIÓN, M. A., P. SÁNCHEZ GÓMEZ, J. GUERRA, A. HERNÁNDEZ GONZÁLEZ, A. F. CARRILLO, J. F. JIMÉNEZ MARTÍNEZ, J. GARCÍA RODRÍGUEZ y J. F. MARTÍNEZ FERNÁNDEZ -2000- Helechos de la región de Murcia. Universidad de Murcia. Murcia. 71 pp.

FABREGAT, C. -1995- Estudio florístico y fitogeográfico de la comarca del Alto Maestrazgo (Castellón), Tesis doctoral. Facultad de Ciencias Biológicas. Universidad de Valencia. inédita.

IBARS, A. M., J. J. HERRERO-BORGOÑÓN, E. ESTRELLES e I. MARTÍNEZ SOLÍS -1999Helechos de la Comunidad Valenciana. Generalitat Valenciana. Conselleria de Medio Ambiente. Valencia. 163 pp.

LÓPEZ GONZÁLEZ, G. -1986- in S. CASTROVIEJO et al. (eds.). Ophioglossum L. Fl. Iber. 1: 35-37. Serv. Publ. CSIC. Real Jardín Botánico. Madrid.

SALVADOR BENEDICTO, J. - 1867- Catálogo de plantas determinadas que se encuentran en los alrededores de Villafranca del Cid, Castellfort, Portell, Ares y parte de Benasal, de la provincia de Castellón (continuación). La Fraternidad 1(22): 427-429. Valencia.
SALVO, A. E., B. CABEZUDO, L. ESPAÑA, T. E. DÍAZ GONZÁLEZ, J. IRANZO, J. IZCO y C. PRADA -1984- Atlas de la pteridoflora ibérica y balear. Acta Bot. Malacitana 9: 105-128.

VIGO, J. -1968- La vegetació del Massís de Penyagolosa. Arxius Secc. Ci. Inst. Estud. Catalans 37. Barcelona. 245 pp.

Aceptado para su publicación en abril de 2001

Dirección de los autores. J. V. Andrés Ros: Jardín Botánico de la Universidad de Valencia, C/ Quart, 80,46008-Valencia; e-mail: jose.v.andres@uv.es. A. J. Castelló Monsoriu: Cno. de Moncada, 276-B, 46025-Valencia: e-mail: braxton@teleline.es. N. Sarasa Alcubierre: Ctra. Almudévar-Alcubierre, s/ n, Robres, 22252-Huesca. 